\title{
Article \\ Acoustic Bessel Vortex Beam by Quasi-Three-Dimensional Reflected Metasurfaces
}

\author{
Yin Wang ${ }^{1,+}$, Jiao Qian ${ }^{1,+}$, Jian-Ping Xia ${ }^{1,+} \mathbb{D}$, Yong Ge ${ }^{1,2}$, Shou-Qi Yuan ${ }^{1}$, Hong-Xiang Sun ${ }^{1,3, *}$ \\ and Xiao-Jun Liu $2,3, *$ (D)
}

1 Research Center of Fluid Machinery Engineering and Technology, School of Physics and Electronic Engineering, School of Computer Science and Communication Engineering, Jiangsu University, Zhenjiang 212013, China; 2112008007@stmail.ujs.edu.cn (Y.W.); jiao.qian@duke.edu (J.Q.); jianping.xia@duke.edu (J.-P.X.); geyong@ujs.edu.cn (Y.G.); shouqiy@ujs.edu.cn (S.-Q.Y.)

2 Collaborative Innovation Center of Advanced Microstructures, Key Laboratory of Modern Acoustics, Department of Physics, Nanjing University, Nanjing 210093, China

3 State Key Laboratory of Acoustics, Institute of Acoustics, Chinese Academy of Sciences, Beijing 100190, China

* Correspondence: jsdxshx@ujs.edu.cn (H.-X.S.); liuxiaojun@nju.edu.cn (X.-J.L.)

+ These authors contributed equally to this work.

Citation: Wang, Y.; Qian, J.; Xia, J.-P.; Ge, Y.; Yuan, S.-Q.; Sun, H.-X.;

Liu, X.-J. Acoustic Bessel Vortex Beam by Quasi-Three-Dimensional Reflected Metasurfaces. Micromachines 2021, 12, 1388. https://doi.org/10.3390/mi12111388

Academic Editors: Adem Ozcelik and Tony Jun Huang

Received: 23 October 2021

Accepted: 9 November 2021

Published: 12 November 2021

Publisher's Note: MDPI stays neutral with regard to jurisdictional claims in published maps and institutional affiliations.

Copyright: (c) 2021 by the authors. Licensee MDPI, Basel, Switzerland. This article is an open access article distributed under the terms and conditions of the Creative Commons Attribution (CC BY) license (https:// creativecommons.org/licenses/by/ $4.0 /)$.

\begin{abstract}
Vortex beams have a typical characteristic of orbital angular momentum, which provides a new degree of freedom for information processing in remote communication and a form of noncontact manipulation for trapping particles. In acoustics, vortex beams are generally observed on the surface of a metamaterial structure or in a waveguide with a hard boundary owing to the characteristic of easy diffusion in free space. The realization of an acoustic vortex beam with a long-distance propagation in free space still remains a challenge. To overcome this, we report a type of acoustic Bessel vortex (ABV) beam created by a quasi-three-dimensional reflected metasurface in free space based on phase modulation. By using the Bessel and vortex phase profiles, we can realize an $\mathrm{ABV}$ beam with the high performances of both Bessel and vortex beams, and its effective propagation distance is larger than $9.2 \lambda$ in free space. Beyond that, we discuss the bandwidth and topological charge of the ABV beam in detail, and the fractional bandwidth can reach about 0.28 . The proposed ABV beam has the advantages of a high-performance vortex, long-distance propagation, and broad bandwidth, which provide a new pathway for designing multifunctional vortex devices with promising applications.
\end{abstract}

Keywords: acoustic wave; vortex beam; Bessel beam; acoustic metasurfaces; phase modulation

\section{Introduction}

Recent years have witnessed the great development of acoustic vortex beams owing to their extensive applications in a wide range of fields, such as sound communication [1,2] and particle trapping [3-5]. The acoustic vortex beam can transfer different-order vortex wavefronts with orbital angular momentum, opening up a new degree of freedom for sound modulations [6-10]. Traditionally, by designing active sound arrays composed of phased sources [2,11-13], researchers have experimentally realized acoustic vortex beams to manipulate micro-particles underwater. However, the high cost and complex circuits of the active systems create some difficulties for their further applications.

To overcome these restrictions, the emergence of acoustic metamaterials [14-20] and metasurfaces [21-27] have provided alternative design schemes for passive acoustic lenses of vortex beams. As examples, by designing thickness-gradient structures [28,29] or an acoustic plate with a spiral shaped structure [30-32], the acoustic vortex beam can be observed around structure surfaces. However, their structures must satisfy the geometric characteristics of spiral distribution. On the other hand, by tailoring phase profiles of acoustic metasurfaces, sound energy can also be formed as an acoustic vortex beam based 
on phase modulation [33-37]. For instance, by designing phase profiles of Helmholtz resonators in a circular waveguide, acoustic vortex beams are also observed, and the sound energy of vortex beams can propagate in the waveguide with a hard boundary [34,37]. Furthermore, multifunctional vortex beams of sound can also be obtained by changing phase profiles, such as focusing vortices $[38,39]$ and vortex beams with asymmetric propagation [40], which have potential special applications. Additionally, the finite element method based on the COMSOL Multiphysics software has been introduced to numerically design and optimize different types of acoustic vortex devices, such as unidirectional vortex beams through acoustic Weyl crystal with a topological lattice defect, and a vortex converter composed of an acoustic metagrating in a waveguide [10,37,40], and the corresponding simulated results agree well with the measured ones. However, these aforementioned vortex beams are generally observed on structure surfaces or in waveguides with a hard boundary owing to the characteristic of easy diffusion in free space. The realization of an acoustic vortex beam with long-distance propagation in free space still poses a great challenge.

In our work, we propose a type of quasi-three-dimensional (quasi-3D) reflected metasurface to realize an acoustic Bessel vortex (ABV) beam by phase modulation. Based on the Bessel and vortex phase profiles, we can realize an ABV beam with the advantages of both a high-performance vortex and long-distance propagation in free space, which overcomes the limitations of easy diffusion in free space. Moreover, we discuss the working bandwidth of the ABV beam in detail, and its fractional bandwidth can reach about 0.28 , showing the characteristic of broad bandwidth. Finally, we design and realize ABV beams with different topological charges. The designed ABV beams with a high-performance vortex and long-distance propagation may lead to important advances in diverse applications.

\section{Design and Methods}

As schematically shown in Figure 1a, we propose a type of quasi-3D reflected unit composed of a cuboid solid with a groove on the upper side. The cross sections $x-z$ and $y-z$ of the unit are the same, and the structure parameters are selected as $w=1.0 \mathrm{~cm}, h=2.5 \mathrm{~cm}$, and $d=0.1 \mathrm{~cm}$. The groove depth $h_{0}$ is tunable to modulate reflected phase delays of sound. The incident wavelength $\lambda$ of sound is selected as $3.8 \mathrm{~cm}$ (viz., frequency $f_{0}=9.0 \mathrm{kHz}$ in air). Throughout this work, the characteristics of sound reflections are numerically simulated by the COMSOL Multiphysics software. Here, the pressure field is calculated in the acoustic-solid interaction module, and the solid structure is made of epoxy resin to satisfy the acoustic-structure boundary conditions. The material parameters are adopted as follows: the density $\rho=1180 \mathrm{~kg} / \mathrm{m}^{3}$, the longitudinal wave velocity $c_{1}=2720 \mathrm{~m} / \mathrm{s}$, and the transversal wave velocity $c_{\mathrm{t}}=1460 \mathrm{~m} / \mathrm{s}$ for epoxy resin; $\rho=1.21 \mathrm{~kg} / \mathrm{m}^{3}$ and the sound velocity $c=343 \mathrm{~m} / \mathrm{s}$ for air.

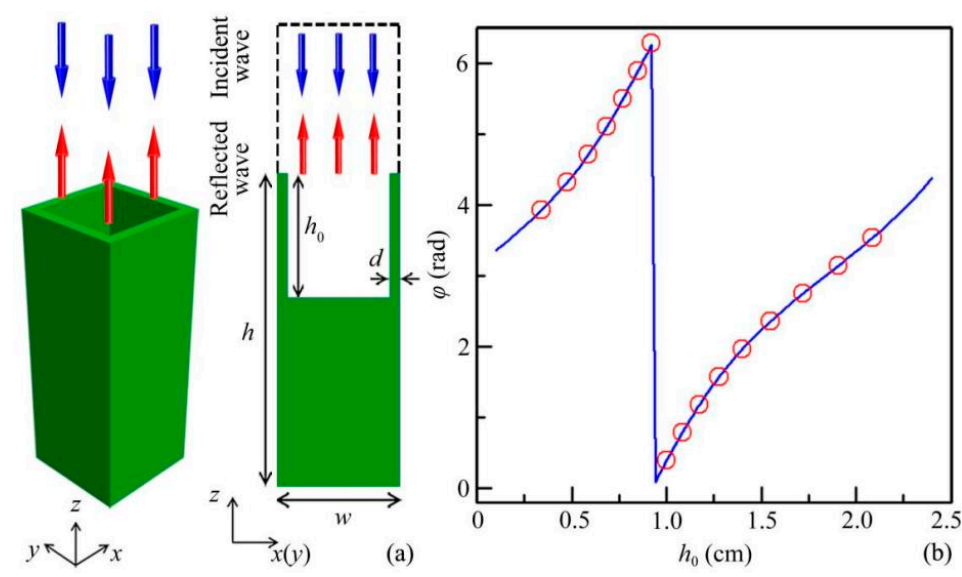

Figure 1. Design of a quasi-3D reflected unit. (a) Schematic of a quasi-3D reflected unit and its $x(y)-z$ cross section. (b) Reflected phase delays created by the quasi-3D reflected unit as a function of tunable depth $h_{0}$. 
Figure $1 \mathrm{~b}$ shows the reflected phase delays of sound created by the quasi-3D units with different values of $h_{0}$. We can see that, in the range $0.1<h_{0}<2.4 \mathrm{~cm}$, the theoretical reflected phase delay (blue solid line) can cover the whole $2 \pi$ range. Here, we select 16 discrete values of $h_{0}$ (red open circles) to realize an equally spaced phase delay from 0 to $2 \pi$ with a step of $\pi / 8$.

Based on the selected 16 reflected phased units, we can design a type of planar quasi$3 \mathrm{D}$ reflected metasurface of the ABV beam by phase modulation. Figure 2a shows the normal incidence of an acoustic wave on the quasi-3D reflected metasurface, and the sound reflections are formed as an ABV beam. To design it, we introduce the phase profile of a vortex beam in free space, which is expressed as

$$
\varphi_{\mathrm{s}}(\theta)=n \theta,
$$

where $n$ is the topological charge of the vortex beam [31], and $\theta$ is the azimuth angle which is in the range from 0 to $2 \pi$ in the $x-y$ plane. To realize a vortex beam with long-distance propagation in free space, we here introduce a non-diffracting Bessel phase profile. Based on the 3D generalized Snell's law [41] and Equation (1), the final phase profile of the designed quasi-3D reflected metasurface in the $x-y$ plane is given as

$$
\varphi=-k \sin \alpha \sqrt{x^{2}+y^{2}}+n \theta
$$

where $k=2 \pi f / c$ is the wave number in air, and $c$ and $f$ are the sound velocity and the frequency, respectively. $\alpha$ is the base angle of the Bessel beam, and the parameter $\theta$ satisfies $\tan \theta=x / y$. Here, the parameters $\alpha$ and $n$ are selected as $15^{\circ}$ and 1 , respectively, and the size of the quasi-3D metasurface in the $x-y$ plane is $40 \mathrm{~cm} \times 40 \mathrm{~cm}$. Figure $2 \mathrm{~b}, \mathrm{c}$ show the theoretical continuous and discrete phase delays of the quasi-3D reflected metasurface in the $x-y$ plane, respectively. It is worth noting that the phase distribution shows characteristics typical of a vortex beam, and both types of phase profiles agree well with each other.
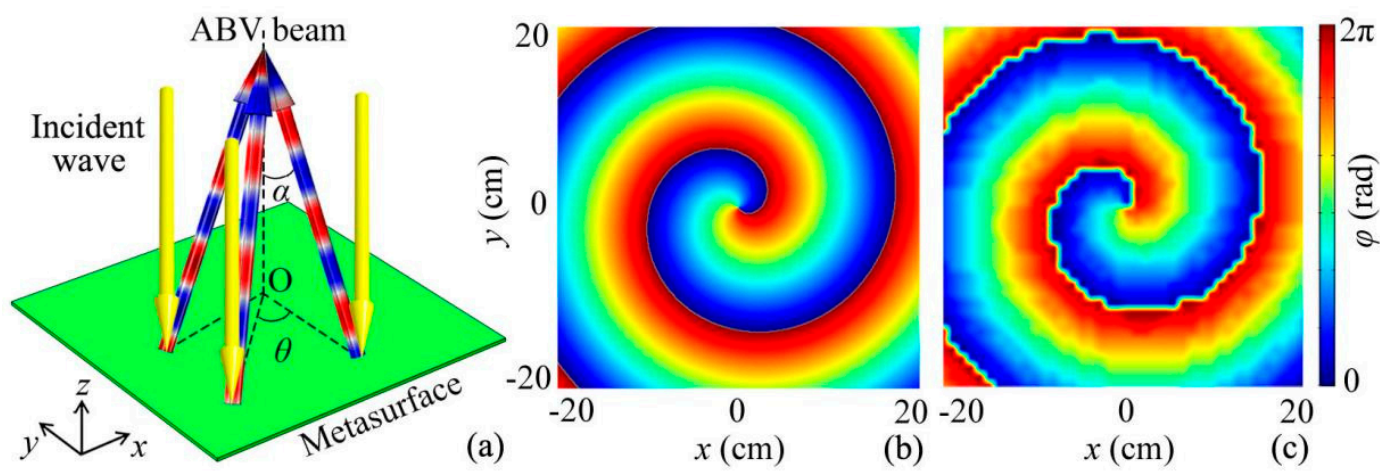

Figure 2. Design of the reflected ABV beam. (a) Schematic of a quasi-3D reflected metasurface of ABV beam, and its (b) theoretical continuous and (c) discrete phase profiles.

\section{Results and Discussion}

\subsection{Performance of $A B V$ Beam}

Based on the two types of phase profiles, we realized an ABV beam by designing a quasi-3D reflected metasurface. Figure 3a shows the simulated 3D reflected intensity distribution created by the designed quasi-3D metasurface composed of 16 types of phased units at $9.0 \mathrm{kHz}$. We observed that the sound reflections were concentrated into a longdistance hollow cylinder along the $z$ direction, which can cover a range from 28 to $72 \mathrm{~cm}$ $\left(|p|^{2}>5.0 \mathrm{~Pa}^{2}\right)$. On the other hand, the energy of the sound beam in the central region was almost zero, showing typical features of both Bessel and vortex beams. To further show the characteristics of the ABV beam, we also displayed the reflected acoustic intensity 
and phase distributions of the ABV beam at 5 selected cross sections $(z=30,40,50,60$, and $70 \mathrm{~cm}$ ), which are shown in Figure $3 b, c$, respectively. It is obvious that, with the increase of $z$, there existed a concentric ring-shaped region at the center of each cross section (Figure $3 \mathrm{~b}$ ), which was the same as that in Figure 3a. Beyond that, the sound energy in the ring-shaped region $\left(|p|^{2}>6.0 \mathrm{~Pa}^{2}\right)$ was much larger than the central energy of sound $\left(|p|^{2} \approx 0\right)$ at the 5 cross sections owing to the existence of a phase singularity at the center (Figure 3c). The phase distributions in Figure 3c show the typical characteristics of the vortex beam with $n=1$, further verifying that the ABV beam had both advantages of long-distance propagation and a high-performance vortex in free space. In addition to the aforementioned results, we simulated the acoustic intensity and phase distributions created by the theoretical continuous phase profile for comparison, which are shown in Figure $3 \mathrm{~d}-\mathrm{f}$. We can see that the characteristics of the observed ABV beam matched well with those in Figure $3 \mathrm{a}-\mathrm{c}$, which further demonstrates the performance of the reflected $\mathrm{ABV}$ beam by the quasi-3D metasurface.
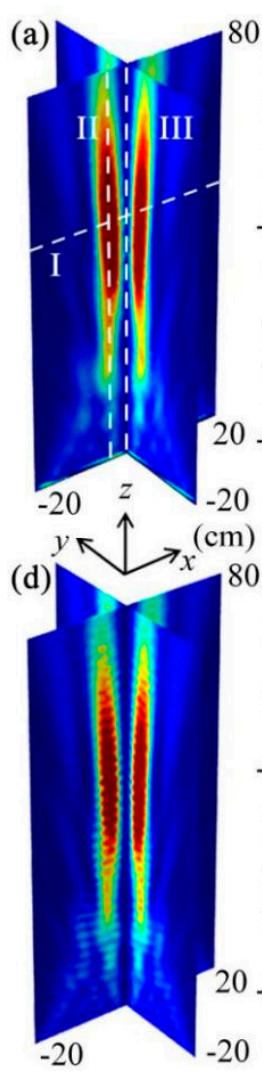

$z=30$
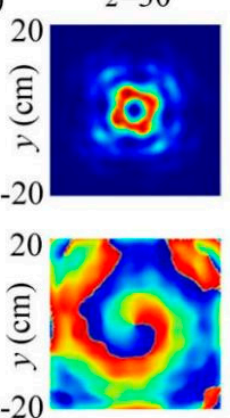

$-20$ $z=40$
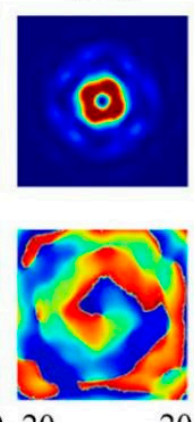

$20-20$ $z=50$
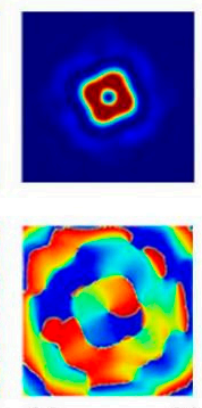

$x(\mathrm{~cm})$

$z=50$
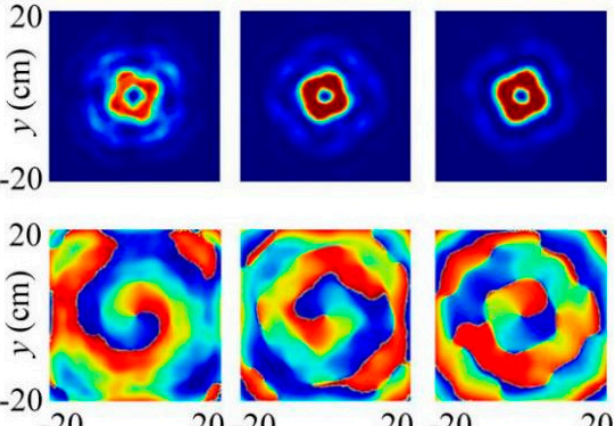
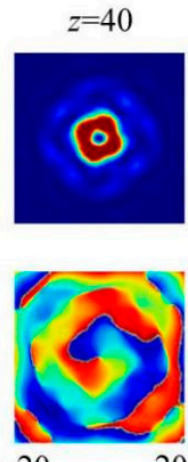

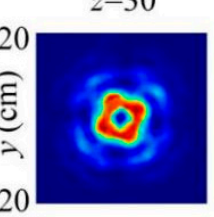

$-20$

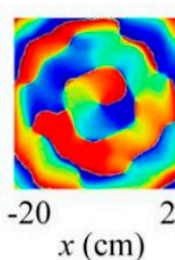

$20-20$

$20-20$

$20-20$

$z=60$
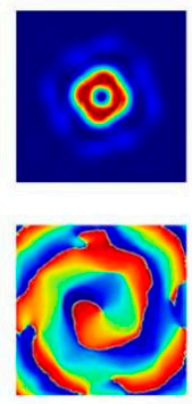

$20-20$

$z=60$
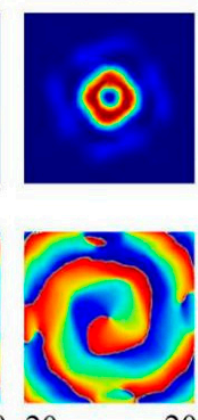

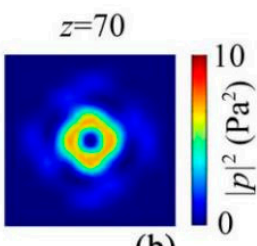

(b)

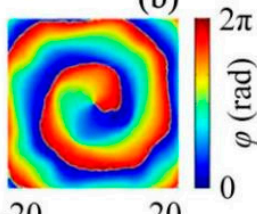

(c)

Figure 3. The performance of the reflected ABV beam with $n=1$ at $9.0 \mathrm{kHz}$. (a) Simulated 3D reflected intensity distribution of the ABV beam created by the quasi-3D metasurface, and its corresponding reflected (b) intensity and (c) phase distributions at 5 selected cross sections $z=30,40,50,60$, and $70 \mathrm{~cm}$. (d) Simulated 3D reflected intensity distribution of the ABV beam created by the theoretical continuous phase profile, and its corresponding reflected (e) intensity and (f) phase distributions at the 5 cross sections.

To quantify the performances of the ABV beam, we also display the simulated longitudinal and transverse intensity distributions of the ABV beam (the lines I-III in Figure 3a), which are shown in Figure 4. We observe that two acoustic energy peaks $\left(|p|^{2} \approx 12.0 \mathrm{~Pa}^{2}\right)$ existed on both sides of $x=0$, while the sound intensity at $x=0$ was close to zero (Figure $4 \mathrm{a}$ ). In addition, as shown in Figure $4 \mathrm{~b}$, the sound intensities along line II were much larger than those along line III $\left(|p|^{2} \approx 0\right)$, which arose from the existence of the phase singularity along line III. Moreover, the total width at half maximum of the sound intensity along line II reached about $35 \mathrm{~cm}(9.2 \lambda)$, showing that the energy of the ABV beam can propagate a 
long distance in free space. Therefore, we further verified the high performances of both Bessel and vortex beams for the ABV beam created by the quasi-3D reflected metasurface.
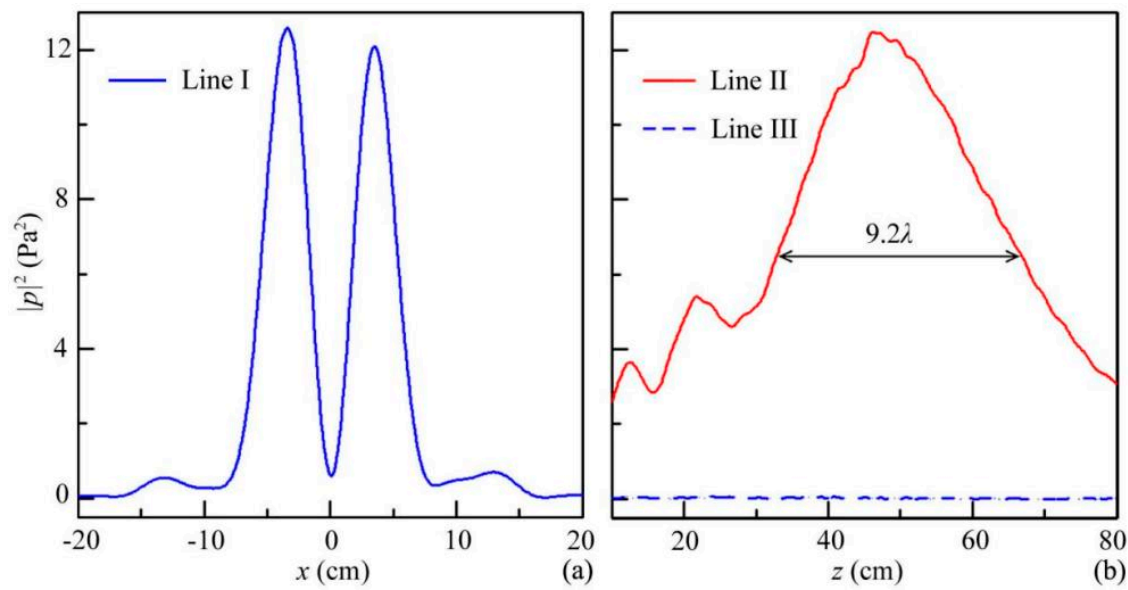

Figure 4. Simulated acoustic intensity distributions through the ABV beam along lines (a) I, (b) II, and III in Figure 3d.

\subsection{Bandwidth of $A B V$ Beam}

Next, we discuss the bandwidth of the quasi-3D reflected metasurface of the ABV beam. Figure 5 shows the intensity and phase distributions created by the quasi-3D reflected metasurface at different frequencies, in which the other parameters remain unchanged. It is noted that, with the increase of frequency, the center position of the ABV beam moved along the $+z$ direction gradually. However, the characteristics of the ABV beam stayed the same as those in Figure $3 \mathrm{~d}-\mathrm{f}$. Moreover, we also simulated the intensity and phase distributions of the ABV beam at both edge frequencies $(8.0$ and $11.0 \mathrm{kHz})$, which are shown in Section Note 1 of the Supplementary Materials. Therefore, the working bandwidth of the designed ABV beam can exceed $2.5 \mathrm{kHz}$, and its fractional bandwidth is larger than 0.28 , showing a broadband characteristic.

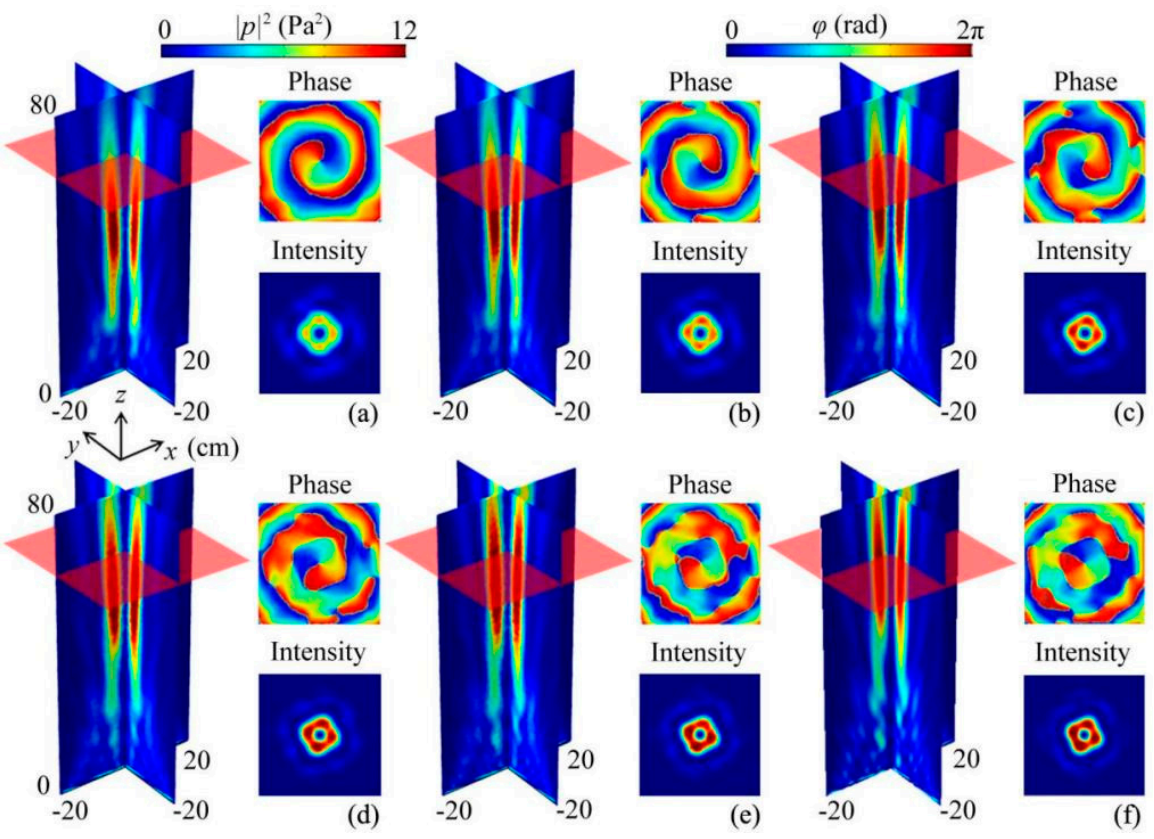

Figure 5. Simulated 3D reflected intensity distributions of the ABV beam created by the quasi-3D metasurface at (a) 8.2, (b) 8.7, (c) 9.2, (d) 9.7, (e) 10.2, and (f) $10.7 \mathrm{kHz}$. Insets on the right side represent the phase and intensity distributions at a cross section $z=60 \mathrm{~cm}$ (red translucent planes). 


\subsection{Design of $A B V$ Beam with Different Values of $n$}

Based on Equation (2), we designed the quasi-3D reflected metasurfaces of the ABV beam with different values of $n$, and their theoretical continuous and discrete phase delays in the $x-y$ plane are shown in Section Note 2 of the Supplementary Materials. Here, we selected $n=2$ as an example, and the simulated intensity and phase distributions created by the quasi-3D reflected metasurface at $9.0 \mathrm{kHz}$, which are shown in Figure 6a,b, respectively. We observe that the sound reflections were concentrated into a hollow cylinder region with a height of $9 \lambda$, and the characteristics of the ABS beam were similar to those in Figure $3 \mathrm{~d}$ $(n=1)$. However, for the phase distribution in Figure $6 \mathrm{~b}$, we observe that the phase delay shifted from 0 to $2 \pi$ twice around in a circle at 3 cross sections $z=20,40$ and $60 \mathrm{~cm}$, which was different from that in Figure $3 \mathrm{f}(n=1)$. Such a phenomenon is also obviously displayed in Figure 6c. Beyond that, we also designed a fractional ABV beam with values of $n=1.25$, 1.5, and 1.75, which is shown in Section Note 3 of the Supplementary Materials. Therefore, by combining both phase profiles of Bessel and vertex beams, we realized an ABV beam with different topological charges.
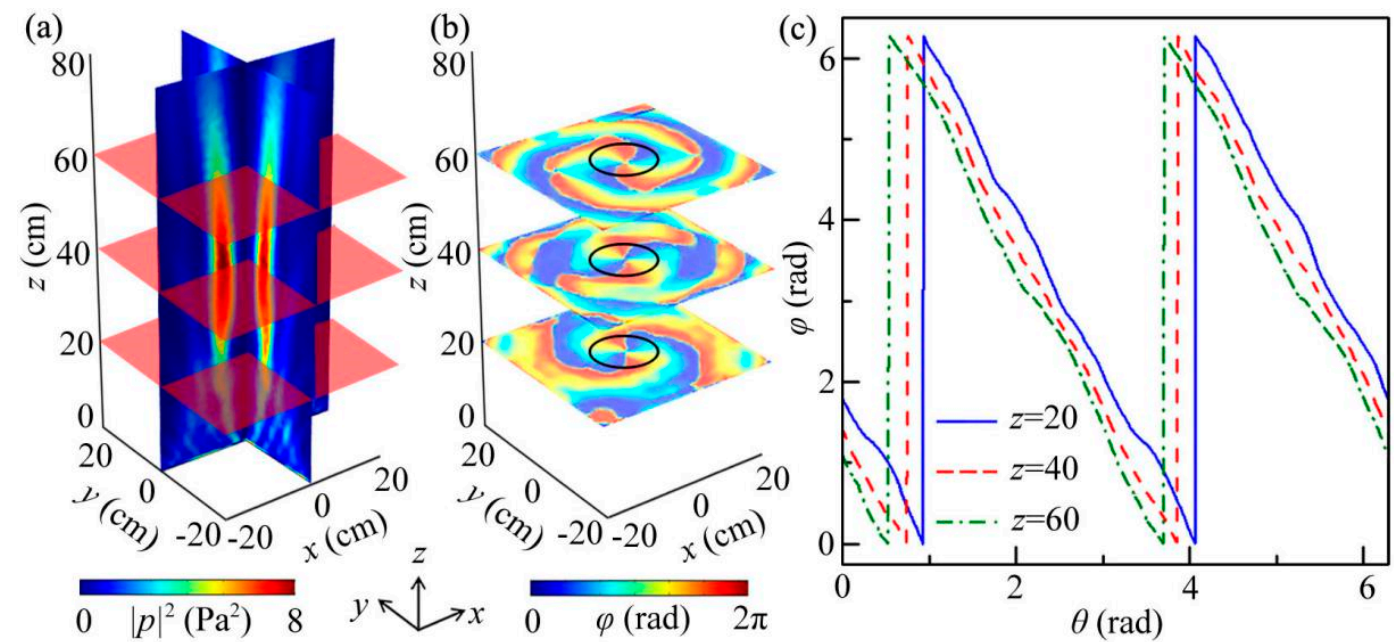

Figure 6. The performance of the reflected ABV beam with $n=2$ at $9.0 \mathrm{kHz}$. (a) Simulated 3D reflected intensity distribution of the ABV beam created by the quasi-3D metasurface, and its corresponding reflected (b) phase distribution at 3 cross sections $z=20,40$, and $60 \mathrm{~cm}$. (c) Phase delays around in a circle with the radius of $6 \mathrm{~cm}$ (black open circles in (b)) as a function of $\theta$ at the 3 cross sections.

\subsection{Design of Underwater ABV Beam}

Finally, we designed a type of planar quasi-3D reflected metasurface of an underwater $\mathrm{ABV}$ beam, and simulated the intensity distribution of the underwater $\mathrm{ABV}$ beam at $30 \mathrm{kHz}$, which is shown in Section Note 4 of the Supplementary Materials. The results show that the simulated underwater ABV beam also exhibited characteristics typical of a vortex beam, which can cover a range from 30 to $120 \mathrm{~cm}\left(|p|^{2}>2.5 \mathrm{~Pa}^{2}\right)$ along the $z$ direction. The propagation distance of the underwater ABV beam (about 22 $\lambda$ ) was larger than that (about $10 \lambda$ ) of the holographic algorithm [42]. The designed underwater ABV beam with longdistance propagation will be important for realizing advanced in vivo acoustic tweezers, as it can penetrate deeper into a lesion location, which can be potentially applied to removing broken bones, thrombus ablation, and nerve stimulation.

\section{Conclusions}

In conclusion, we have demonstrated a quasi-3D reflected metasurface of an ABV beam. The results show that, by using the Bessel and vortex phase profiles, we realized an $\mathrm{ABV}$ beam with a high-performance vortex, and its effective propagation distance can exceed $9.2 \lambda$ in free space, which overcomes the restriction of easy diffusion of vortex beams propagating in free space. Additionally, the designed ABV beam had the characteristic of 
broad bandwidth, and its fractional bandwidth reached about 0.28 . Finally, by modulating the phase profile of the quasi-3D reflected metasurface, we observed ABV beams with different topological charges, where $n=1.25,1.5,1.75$, and 2 . In contrast to the characteristics of acoustic vortex beams [31,34], the proposed ABV beam with the features of long-distance propagation, a high-performance vortex and broad bandwidth provides a novel methodology for sound manipulations and a fertile ground for designing multifunctional vortex devices, which also play an important role in the field of underwater structure measurement.

Supplementary Materials: The following are available online at https:/ /www.mdpi.com/article/10

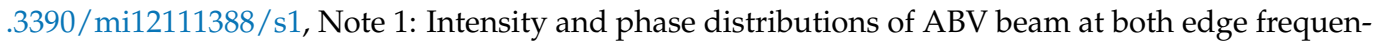
cies. Note 2: Theoretical continuous and discrete phase delays of the quasi-3D reflected metasurface with different values of $n$. Note 3: Intensity and phase distributions of fractional reflected ABV beam. Note 4: Design of underwater ABV Beam

Author Contributions: Conceptualization, Y.W. and J.-P.X.; methodology, Y.W. and J.Q.; validation, Y.G.; formal analysis, H.-X.S. and Y.G.; writing-original draft preparation, Y.W.; writing-review and editing, H.-X.S.; supervision, H.-X.S., S.-Q.Y. and X.-J.L. All authors have read and agreed to the published version of the manuscript.

Funding: This work was supported by the National Natural Science Foundation of China (Grant Nos. 11774137, 11834008, 12174159, and 51779107), the Postgraduate Research and Practice Innovation Program of Jiangsu Province (Grant No. KYCX20_3072), and the Undergraduate Practice Innovation Program of Industrial Center in Jiangsu University (Grant No. ZXJG2019105).

Conflicts of Interest: The authors declare no conflict of interest.

\section{References}

1. Brunet, T.; Thomas, J.L.; Marchiano, R. Transverse shift of helical beams and subdiffraction imaging. Phys. Rev. Lett. 2010, 105, 034301. [CrossRef]

2. Shi, C.; Dubois, M.; Wang, Y.; Zhang, X. High-speed acoustic communication by multiplexing orbital angular momentum. Proc. Natl. Acad. Sci. USA 2017, 114, 7250-7253. [CrossRef]

3. Baresch, D.; Thomas, J.L.; Marchiano, R. Observation of a single-beam gradient force acoustical trap for elastic particles: Acoustical tweezers. Phys. Rev. Lett. 2016, 116, 024301. [CrossRef]

4. Marzo, A.; Ghobrial, A.; Cox, L.; Caleap, M.; Croxford, A.; Drinkwater, B.W. Realization of compact tractor beams using acoustic delay-lines. Appl. Phys. Lett. 2017, 110, 014102. [CrossRef]

5. Ren, X.; Zhou, Q.; Xu, Z.; Liu, X. Particle trapping in arbitrary trajectories using first-order bessel-Like acoustic beams. Phys. Rev. Appl. 2021, 15, 054041. [CrossRef]

6. Strahan, S.E.; Oman, L.D.; Douglass, A.R.; Coy, L. Modulation of antarctic vortex composition by the quasi-biennial oscillation. Geophys. Res. Lett. 2015, 42, 4216-4223. [CrossRef]

7. Gong, Z.; Baudoin, M. Particle assembly with synchronized acoustic tweezers. Phys. Rev. Appl. 2019, 12, 024045. [CrossRef]

8. Liu, Y.; Zhang, X.; Guo, J.; Yang, H.; Han, L.; Yao, Y.; Wu, F. Tailoring of diversified sound vortices using curved impedancematched acoustic metasurfaces. Mod. Phys. Lett. B 2020, 34, 2050121. [CrossRef]

9. Zhang, H.; Zhang, W.; Liao, Y.; Zhou, X.; Li, J.; Hu, G.; Zhang, X. Creation of acoustic vortex knots. Nat. Commun. 2020, 11, 1-6. [CrossRef]

10. Wang, Q.; Ge, Y.; Sun, H.X.; Xue, H.R.; Jia, D.; Guan, Y.J.; Yuan, S.Q.; Zhang, B.L.; Chong, Y.D. Vortex states in an acoustic Weyl crystal with a topological lattice defect. Nat. Commun. 2021, 12, 1-8. [CrossRef]

11. Marchiano, R.; Thomas, J.-L. Synthesis and analysis of linear and nonlinear acoustical vortices. Phys. Rev. E 2005, 71, 066616. [CrossRef] [PubMed]

12. DeMore, C.E.M.; Yang, Z.; Volovick, A.; Cochran, S.; MacDonald, M.; Spalding, G. Mechanical evidence of the orbital angular momentum to energy ratio of vortex beams. Phys. Rev. Lett. 2012, 108, 194301. [CrossRef] [PubMed]

13. Riaud, A.; Thomas, J.L.; Charron, E.; Bussonnière, A.; Matar, O.B.; Baudoin, M. Anisotropic swirling surface acoustic waves from inverse filtering for on-chip generation of acoustic vortices. Phys. Rev. Appl. 2015, 4, 034004. [CrossRef]

14. Liang, Z.; Li, J. Extreme acoustic metamaterial by coiling up space. Phys. Rev. Lett. 2012, 108, 114301. [CrossRef]

15. Cummer, S.A.; Christensen, J.; Alù, A. Controlling sound with acoustic metamaterials. Nat. Rev. Mater. 2016, 1, 16001. [CrossRef]

16. Wu, Y.; Yang, M.; Sheng, P. Perspective: Acoustic metamaterials in transition. J. Appl. Phys. 2018, 123, 090901. [CrossRef]

17. Fan, X.D.; Zou, Z.; Zhang, L. Acoustic vortices in inhomogeneous media. Phys. Rev. Res. 2019, 1, 032014. [CrossRef]

18. Li, J.; Díaz-Rubio, A.; Shen, C.; Jia, Z.; Tretyakov, S.; Cummer, S. Highly efficient generation of angular momentum with cylindrical bianisotropic metasurfaces. Phys. Rev. Appl. 2019, 11, 024016. [CrossRef] 
19. Cheng, Y.Z.; Li, W.Y.; Mao, X.S. Triple-band polarization angle independent $90^{\circ}$ polarization rotator based on fermat's spiral structure planar chiral metamaterial. Prog. Electromagn. Res. 2019, 165, 35. [CrossRef]

20. Ma, F.; Chen, J.; Wu, J.H. Experimental study on performance of time reversal focusing. J. Phys. D Appl. Phys. 2019, 53, 055302. [CrossRef]

21. Li, Y.; Liang, B.; Gu, Z.M.; Zou, X.Y.; Cheng, J.C. Reflected wavefront manipulation based on ultrathin planar acoustic metasurfaces. Sci. Rep. 2013, 3, srep02546. [CrossRef] [PubMed]

22. Mei, J.; Wu, Y. Controllable transmission and total reflection through an impedance-matched acoustic metasurface. New J. Phys. 2014, 16, 123007. [CrossRef]

23. Xia, J.P.; Zhang, X.T.; Sun, H.X.; Yuan, S.Q.; Qian, J.; Ge, Y. Broadband tunable acoustic asymmetric focusing lens from dual-layer metasurfaces. Phys. Rev. Appl. 2018, 10, 014016. [CrossRef]

24. Gao, N.; Lu, K. An underwater metamaterial for broadband acoustic absorption at low frequency. Appl. Acoust. 2020, 169, 107500. [CrossRef]

25. Zhou, H.T.; Fan, S.W.; Li, X.S.; Fu, W.X.; Wang, Y.F.; Wang, Y.S. Tunable arc-shaped acoustic metasurface carpet cloak. Smart Mater. Struct. 2020, 29, 065016. [CrossRef]

26. Allen, K.W.; Dykes, D.J.P.; Reid, D.R.; Lee, R.T. Multi-objective genetic algorithm optimization of frequency selective metasurfaces to engineer Ku-passband filter responses. Prog. Electromagn. Res. 2020, 167, 19-30. [CrossRef]

27. Jiménez, N.; Groby, J.P.; Romero-García, V. Spiral sound-diffusing metasurfaces based on holographic vortices. Sci. Rep. 2021, 11, 1-13. [CrossRef] [PubMed]

28. Wunenburger, R.; Lozano, J.I.V.; Brasselet, E. Acoustic orbital angular momentum transfer to matter by chiral scattering. New J. Phys. 2015, 17, 103022. [CrossRef]

29. Jiang, X.; Shi, C.; Wang, Y.; Smalley, J.; Cheng, J.; Zhang, X. Nonresonant metasurface for fast decoding in acoustic communications. Phys. Rev. Appl. 2020, 13, 014014. [CrossRef]

30. Wang, T.; Ke, M.; Li, W.; Yang, Q.; Qiu, C.; Liu, Z. Particle manipulation with acoustic vortex beam induced by a brass plate with spiral shape structure. Appl. Phys. Lett. 2016, 109, 123506. [CrossRef]

31. Jia, Y.R.; Wei, Q.; Wu, D.J.; Xu, Z.; Liu, X.J. Generation of fractional acoustic vortex with a discrete archimedean spiral structure plate. Appl. Phys. Lett. 2018, 112, 173501. [CrossRef]

32. Jiménez, N.; Romero-García, V.; García-Raffi, L.M.; Camarena, F.; Staliunas, K. Sharp acoustic vortex focusing by Fresnel-spiral zone plates. Appl. Phys. Lett. 2018, 112, 204101. [CrossRef]

33. Ye, L.; Qiu, C.; Lu, J.; Tang, K.; Jia, H.; Ke, M.; Peng, S.; Liu, Z. Making sound vortices by metasurfaces. AIP Adv. 2016, 6, 085007. [CrossRef]

34. Jiang, X.; Li, Y.; Liang, B.; Cheng, J.C.; Zhang, L. Convert acoustic resonances to orbital angular momentum. Phys. Rev. Lett. 2016, 117, 034301. [CrossRef] [PubMed]

35. Zhang, Y.; Xie, B.; Liu, W.; Cheng, H.; Chen, S.; Tian, J. Anomalous reflection and vortex beam generation by multi-bit coding acoustic metasurfaces. Appl. Phys. Lett. 2019, 114, 091905. [CrossRef]

36. Luo, Y.; Jia, Y.; Yao, J.; Wu, D.; Liu, X. Enhanced fractional acoustic vortices by an annulus acoustic metasurface with multi-layered rings. Adv. Mater. Technol. 2020, 5, 2000356. [CrossRef]

37. Long, Y.; Zhang, D.; Yang, C.; Ge, J.; Chen, H.; Ren, J. Realization of acoustic spin transport in metasurface waveguides. Nat. Commun. 2020, 11, 1-7. [CrossRef]

38. Chen, D.-C.; Zhou, Q.X.; Zhu, X.-F.; Xu, Z.; Wu, D.-J. Focused acoustic vortex by an artificial structure with two sets of discrete Archimedean spiral slits. Appl. Phys. Lett. 2019, 115, 083501. [CrossRef]

39. Guo, S.; Guo, X.; Wang, X.; Du, X.; Wu, P.; Bouakaz, A.; Wan, M. Manipulation of nanodroplets via a nonuniform focused acoustic vortex. Phys. Rev. Appl. 2020, 13, 034009. [CrossRef]

40. Fu, Y.; Shen, C.; Zhu, X.; Li, J.; Liu, Y.; Cummer, S.A.; Xu, Y. Sound vortex diffraction via topological charge in phase gradient metagratings. Sci. Adv. 2020, 6, 9876. [CrossRef]

41. Qian, J.; Wang, Y.; Yuan, S.Q.; Sun, H.X.; Liu, X.J. Reflected acoustic wavefront manipulation by an ultrathin metasurface based on three-dimensional generalized Snell's law. Appl. Phys. Express 2019, 12, 094001. [CrossRef]

42. Liu, D.L.; Waag, R. Propagation and backpropagation for ultrasonic wavefront design. IEEE Trans. Ultrason. Ferroelectr. Freq. Control. 1997, 44, 1-13. [CrossRef] [PubMed] 